\title{
Successful Treatment of Cerebral Sinus Thrombosis with Edox- aban Alone
}

\author{
Yasushi Shibata*
}

Department of Neurosurgery, Mito Medical Center, University of Tsukuba, Ibaraki, Japan

*Corresponding author: Yasushi Shibata, MD, PhD, Department of Neurosurgery, Mito Medical Center, University of Tsukuba, Ibaraki, Japan, E-mail: yshibata@md.tsukuba.ac.jp

\begin{abstract}
As initial therapy for cerebral sinus thrombosis, heparin injection and subsequent oral anticoagulant, namely warfarin, has been recommended. We treated a patient with cerebral sinus thrombosis with edoxaban alone as an outpatient. This is the first report of cerebral sinus thrombosis being treated with edoxaban alone. For patients with mild cerebral venous or sinus thrombosis without severe neurological symptoms, treatment with a DOAC at an outpatient clinic is more effective in ensuring the patient's QOL and reducing medical costs.
\end{abstract}

\section{Keywords}

Cerebral sinus thrombosis, DOACs, Edoxaban

\section{Introduction}

Cerebral sinus thrombosis is a relatively rare disease. Because the most frequent initial symptom is non-specific headache, the early diagnosis of cerebral sinus thrombosis is not easy. However, any delay in the diagnosis of cerebral sinus thrombosis is accompanied by severe symptom caused by cerebral damage and a poor prognosis.

As initial therapy for cerebral sinus thrombosis, heparin injection and subsequent oral anticoagulant, namely warfarin, has been recommended [1]. Recently, Direct Oral Anticoagulants (DOACs) have demonstrated the same efficacy and better safety than warfarin for patients with deep venous thrombosis [2-4]. The clinical effects and risks of DOACs for patients with cerebral venous or sinus thrombosis have not been established; however, better efficacy and safety than warfarin are expected. Indeed, there are some case reports of cerebral sinus thrombosis treated with dabigatran and riva- roxaban describing good efficacy and safety.

Edoxaban has good efficacy and low complication for venous thrombosis [4]. One a day intake and same prescription dose as the prevention of cerebral embolism from non-valvular atrial fibrillation are the advantage of edoxaban. We treated a patient with cerebral sinus thrombosis with edoxaban alone as an outpatient. This is the first report of the treatment of cerebral sinus thrombosis with edoxaban alone.

\section{Case Report}

A previously healthy 47-year-old woman visited our hospital complaining of headache for the previous 7 days. She had no remarkable medical history, like surgery, infection, trauma, pregnancy, of medication, except for anemia. She had had symptoms of a common cold and a fever of $38{ }^{\circ} \mathrm{C}$ from 7 days earlier. Her fever disappeared quickly, but her headache did not improve despite taking analgesics.

Physical and neurological examinations showed no abnormalities. Her blood pressure and body temperature were within normal range. Brain Computed Tomography (CT) showed no brain lesion except for high density from the right lateral to sigmoid sinus (Figure 1). Brain Magnetic Resonance (MR) imaging and MR angiography also showed no brain or arterial abnormalities. T1- and T2-weighted images showed isointensity and loss of flow void at the right lateral and sigmoid sinus, and $\mathrm{T}^{*}$ image revealed low intensity thrombosis from the right lateral to sigmoid sinus (Figure 2). MR venography did not demonstrate flow of the right lateral to sigmoid sinus (Figure 3). A blood examination showed anemia

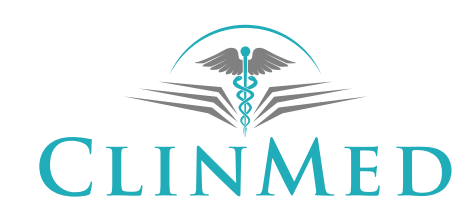

INTERNATIONAL LIBRARY

Citation: Shibata Y (2017) Successful Treatment of Cerebral Sinus Thrombosis with Edoxaban Alone. Int J Crit Care Emerg Med 3:029. doi.org/10.23937/2474-3674/1510029

Received: October 24, 2017: Accepted: November 20, 2017: Published: November 22, 2017

Copyright: (C) 2017 Shibata Y. This is an open-access article distributed under the terms of the Creative Commons Attribution License, which permits unrestricted use, distribution, and reproduction in any medium, provided the original author and source are credited. 

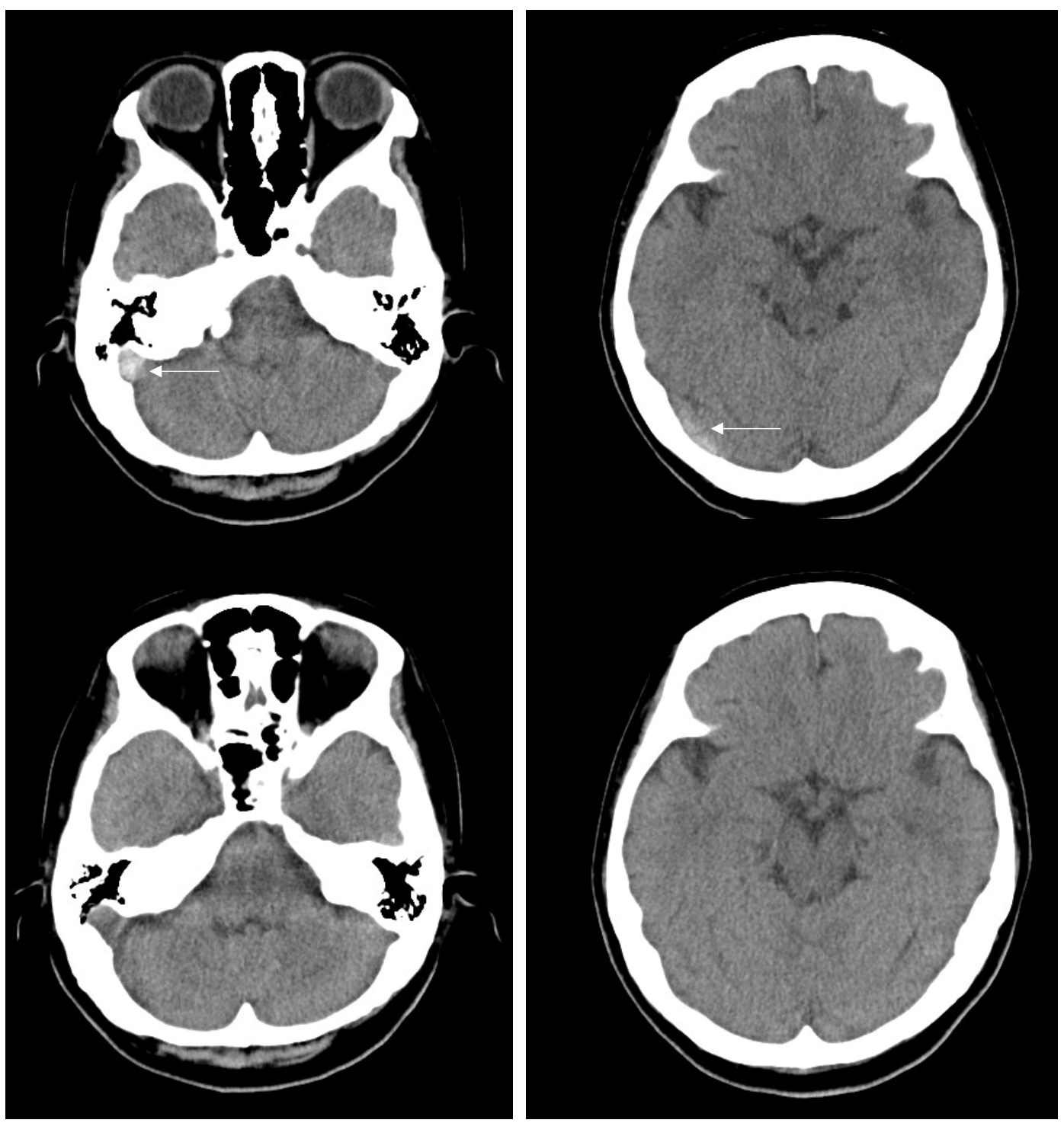

Figure 1: Initial Brain CT (upper) showed high density from the right lateral to sigmoid sinus (arrows). This high density at the sinus disappeared within two weeks after the therapy (lower).

and mild elevation of Fibrinogen Degradation Products (FDP), d-dimer and C-reactive Protein (CRP). Because her only symptom was a headache with no neurological abnormalities and she wished to be treated at an outpatient clinic, we prescribed the DOAC edoxaban at $30 \mathrm{mg}$ once daily. The usual dose of edoxaban for patients with venous thrombosis is $60 \mathrm{mg}$. However, this patient's body weight was less than $50 \mathrm{~kg}$, so a reduced daily dose of $30 \mathrm{mg}$ was selected.

Her headache and high density at the sinus on brain CT disappeared within two weeks after starting edoxaban intake (Figure 1). A blood examination at one month after the start of edoxaban showed normalization of FDP and slight elongation of Prothrombin Time (PT) and Activated Partial Thromboplastin Time (APTT). Brain MR imaging and MR venography at two months after the start of edoxaban demonstrated recanalization of the occluded sinus (Figure 3). The prescription of edoxaban was terminated, and no symptoms have been reported since then.

\section{Discussion}

The indications of DOAC are the prevention of car- diogenic cerebral thrombosis and the prevention and treatment of deep venous thrombosis. The recommended initial treatment of deep venous thrombosis is continuous intravenous infusion of heparin under hospital admission and subsequent oral administration of an anticoagulant [1]. However, the rationale supporting this recommendation is weak. A randomized controlled trial comparing heparin treatment with placebo for cerebral venous thrombosis showed a better outcome for heparin [5]. However, in that study, heparin administration was started late (20-30 days after onset). The study was preliminarily stopped and included only 20 patients. Another randomized controlled trial of 60 patients with cerebral venous thrombosis detected no significant difference between heparin and placebo [6].

Cerebral sinus thrombosis is a rare disease, and its early diagnosis is not easy. Therefore, a large controlled study for cerebral sinus thrombosis may be difficult. Recently several types of DOAC have become available. The non-inferiority of DOACs against heparin plus warfarin for the prevention and treatment of deep venous thrombosis has been reported [2-4]. The clinical efficacy 

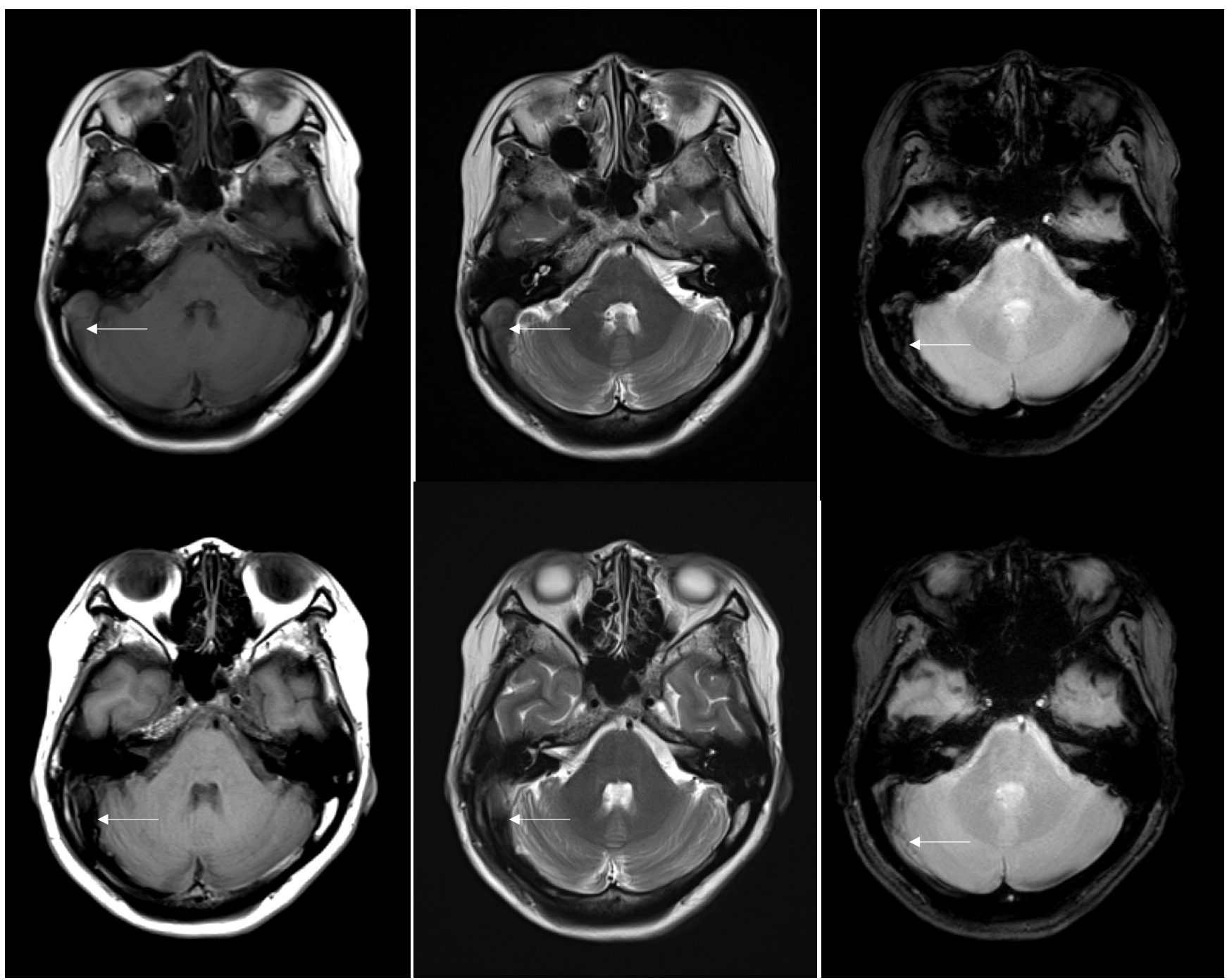

Figure 2: Initial T1 (left) and T2 (middle) weighted images showed isointensity and loss of flow void at the right lateral and sigmoid sinus, and T2* image (Right) revealed low intensity thrombosis from the right lateral to sigmoid sinus (upper). MR imaging at two months after the start of edoxaban demonstrated recanalization of the occluded sinus (lower).

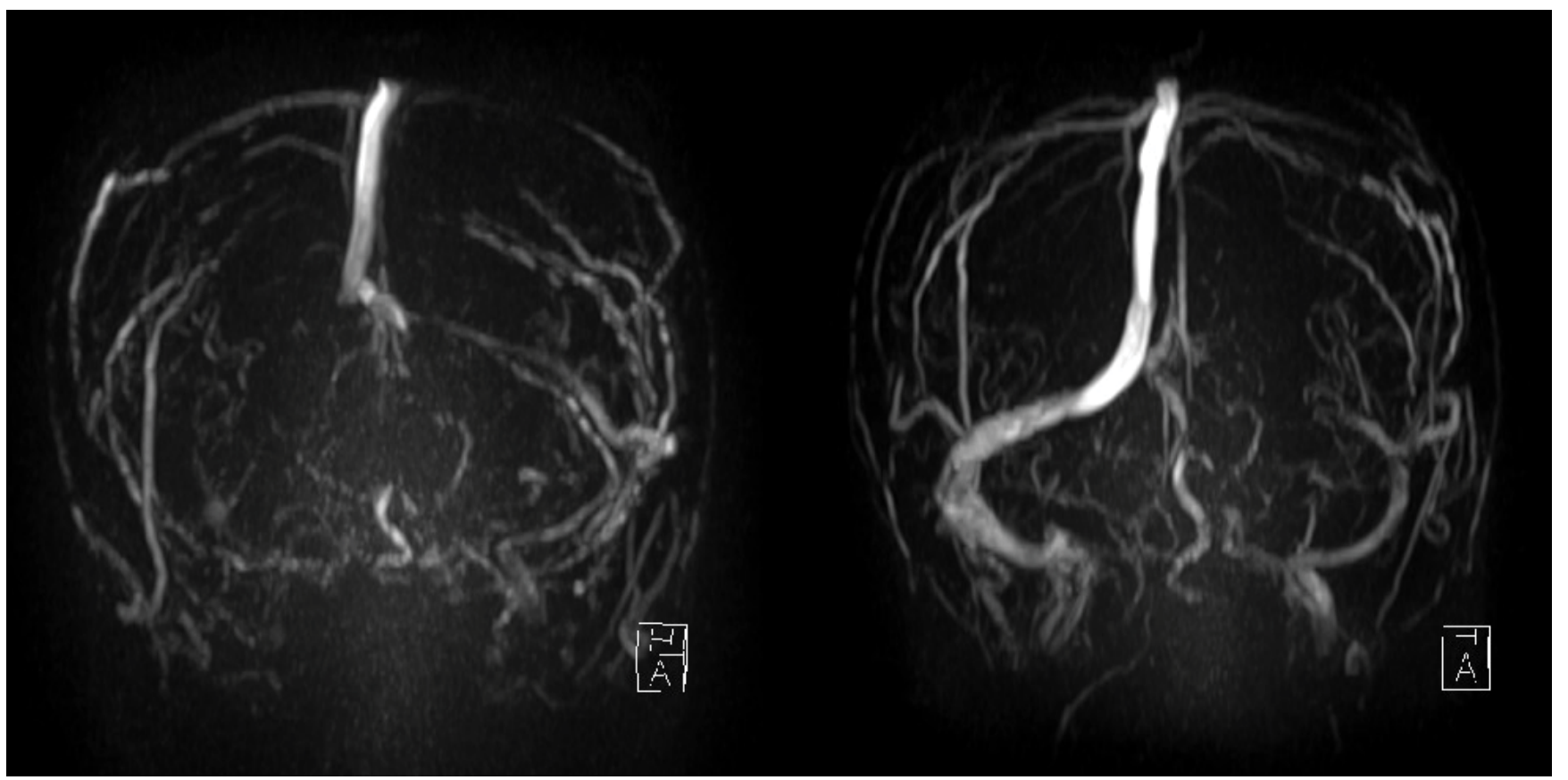

Figure 3: Initial MR venography did not demonstrate flow of the right lateral to sigmoid sinus (left). MR venography at two months after the start of edoxaban demonstrated recanalization of the occluded sinus (right). 
and risks of DOACs for patients with cerebral venous or sinus thrombosis has not been established; however, greater efficacy and safety than with warfarin are expected.

There have been some case reports of cerebral sinus thrombosis being treated with dabigatran, rivaroxaban or edoxaban [7-10]. However, none of these case reports were randomized controlled trials. Most of these reports used heparin as the initial therapy, and dabigatran, rivaroxaban or edoxaban was effective and safe for these patients with cerebral sinus thrombosis.

To our knowledge, our case seems to be the first report of cerebral sinus thrombosis treated with edoxaban alone. We did not select heparin treatment as the initial therapy because the patient wished to be treated at an outpatient clinic. The efficacy and safety of our treatment should be evaluated in a large systematic study. However, because of the rarity of cerebral sinus thrombosis and the difficulty of its early diagnosis, single case reports such as ours may be the only significant contributors to decision-making for clinical practitioners.

The cost of 5000 units of heparin is 2000-3000 Japanese Yen (approximately $\$ 18-27$ US), and continuous heparin intravenous infusion therapy requires hospital admission. The cost of a $30 \mathrm{mg}$ tablet of edoxaban is 748 Japanese Yen (approximately $\$ 6.7$ US), and the drug can be administered on an outpatient basis. Therefore, single-drug treatment with edoxaban at an outpatient clinic can omit hospital admission costs as well as save on medication costs. A propensity-matched cohort study of hemodynamically stable pulmonary embolism patients showed that outpatient treatment was associated with a lower rate of adverse events than hospitalization [11]. During oral outpatient treatment with edoxaban, patients can continue their daily life, so such therapy can help ensure patients' Quality of Life (QOL).

\section{Conclusion}

We treated a patient with cerebral sinus thrombosis with edoxaban alone as an outpatient. This is the first report of cerebral sinus thrombosis being treated with edoxaban alone. For patients with mild cerebral venous or sinus thrombosis without severe neurological symp- toms, treatment with a DOAC at an outpatient clinic is more effective in ensuring the patient's QOL and reducing medical costs.

\section{References}

1. Kernan WN, Ovbiagele B, Black HR, Bravata DM, Chimowitz MI, et al. (2014) Guidelines for the prevention of stroke in patients with stroke and transient ischemic attack: A guideline for healthcare professionals from the American Heart Association/American Stroke Association. Stroke 45: 2160-2236.

2. Prins $M H$, Lensing $A W$, Bauersachs $R$, van Bellen $B$, Bounameaux $H$, et al. (2013) Oral rivaroxaban versus standard therapy for the treatment of symptomatic venous thromboembolism: a pooled analysis of the EINSTEIN-DVT and PE randomized studies. Thromb J 11: 21.

3. Agnelli G, Buller HR, Cohen A, Curto M, Gallus AS, et al. (2013) Oral apixaban for the treatment of acute venous thromboembolism. N Engl J Med 369: 799-808.

4. Buller HR, Decousus H, Grosso MA, Mercuri M, Middeldorp $S$, et al. (2013) Edoxaban versus warfarin for the treatment of symptomatic venous thromboembolism. $\mathrm{N}$ Engl J Med 369: 1406-1415.

5. Einhäupl KM, Villringer A, Mehraein S, Garner C, Pellkofer $M$, et al. (1991) Heparin treatment in sinus venous thrombosis. Lancet 338: 597-600.

6. de Bruijn SF, Stam J (1999) Randomized, Placebo-Controlled Trial of Anticoagulant Treatment With Low-Molecular-Weight Heparin for Cerebral Sinus Thrombosis. Stroke 30: 484-488.

7. Hon SF, Li HL, Cheng PW (2012) Use of Direct Thrombin Inhibitor for Treatment of Cerebral Venous Thrombosis. J Stroke Cerebrovasc Dis 21: 915.e11-915.e15.

8. Geisbüsch C, Richter D, Herweh C, Ringleb PA, Nagel S (2014) Novel Factor Xa Inhibitor for the Treatment of Cerebral Venous and Sinus Thrombosis. Stroke 45: 2469-2471.

9. Mendonça MD, Barbosa R, Cruz-e-Silva V, Calado S, Viana-Baptista M (2015) Oral direct thrombin inhibitor as an alternative in the management of cerebral venous thrombosis: A series of 15 patients. Int J Stroke 10: 1115-1118.

10. Amemiya T, Uesaka T, Kameda K, Kameda K, Uno J, et al. (2017) Usefulness of Edoxaban for Deep Cerebral Venous Sinus Thrombosis with Hemorrhagic Infarction:A Case Report. No Shinkei Geka 45: 607-613.

11. Roy PM, Corsi DJ, Carrier M, Theogene A, de Wit C, et al. (2017) Net clinical benefit of hospitalization versus outpatient management of patients with acute pulmonary embolism. J Thromb Haemost 15: 685-694. 